\title{
Inelastic Neutron Scattering by Gaseous $\mathrm{O}_{2}{ }^{*}$
}

\author{
G. C. SUMMERTIELd AND' N. A. LURIE $\dagger$ \\ Department of Nuclear Engineering, University of Michigan, Ann Arbor, Michigan.
}

(Received 7 March 1968)

\begin{abstract}
The magnetic contributions to neutron diffraction by molecular oxygen have been studied both experimentally and theoretically. We extend the theoretical studies by performing a calculation of the magnetic interaction in the inelastic neutron cross section for gaseous $\mathrm{O}_{2}$. The magnetic inelastic cross section for $\mathrm{O}_{2}$ is significantly different from the nuclear inelastic cross section due to the lack of spherical symmetry in the magnetic-moment distribution about each oxygen nucleus. It is expected from these results that the inelastic cross section for $\mathrm{O}_{2}$ will provide more information about the magnetic-moment distribution than the angular distribution. We present representative calculations to indicate the size and nature of the magnetic contribution to the inelastic cross section.
\end{abstract}

\section{INTRODUCTION}

Neutron diffraction from molecular oxygen has been studied both experimentally ${ }^{1,2}$ and theoretically., ${ }^{3,4}$ The

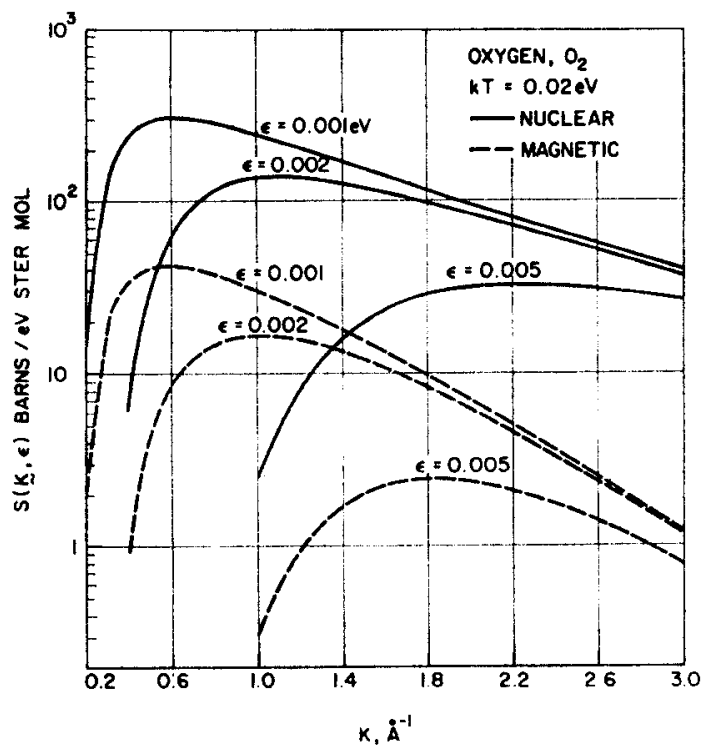

Fig. 1. Nuclear and magnetic contributions to the scattering law for gaseous, molecular oxygen for energy transfers of $\epsilon=0.001$, 0.002 , and $0.005 \mathrm{eV}(a=0.11918 \AA)$.

early gas-diffraction experiments showed the existence of a magnetic contribution due to the paramagnetic nature of the oxygen molecules. Magnetic scattering has also been observed in diffraction from the liquid. ${ }^{2}$ Theoretical analyses of the magnetic scattering in oxygen have been confined to calculations of $d \sigma / d \Omega$. We extend the theoretical studies by presenting detailed

\footnotetext{
* Work supported in part by the U.S. National Science Foundation.

† U.S. Atomic Energy Commission Predoctoral Fellow.

1 H. Palevsky and R. M. Eisberg, Phys. Rev. 98, 492 (1955).

2 D. G. Henshaw, Phys. Rev. 119, 22 (1960).

${ }^{3}$ O. Halpern and G. L. Appleton, Phys. Rev. 90, 869 (1953).

4 W. H. Kleiner, Phys. Rev. 97, 411 (1955)
}

calculations of the inelastic or double differential cross section taking into account both the nuclear and magnetic contributions. The aim of such studies is to learn more about the magnetic-moment distribution which gives rise to the magnetic scattering in oxygen.

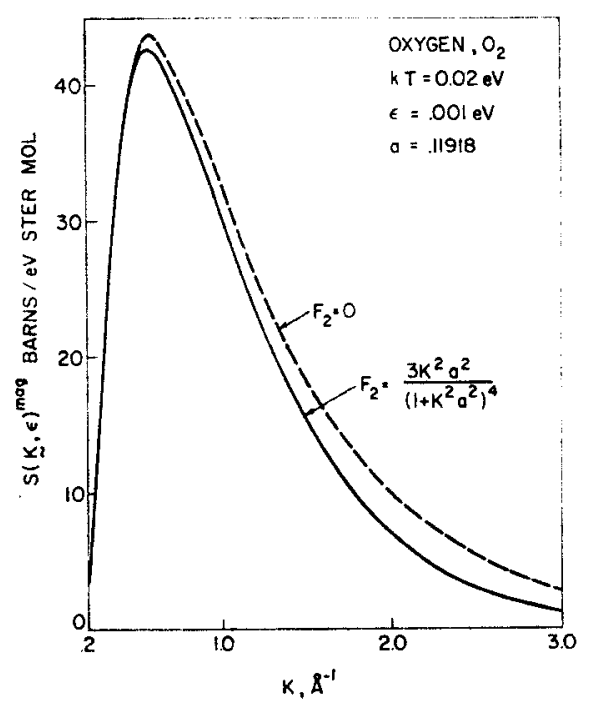

FIG. 2. Magnetic contributions to the scattering law for gaseous, molecular oxygen for two different form factors, $F_{2}=0$ and $F_{2}=$ $3 \kappa^{2} a^{2} /\left(1+\kappa^{2} a^{2}\right)^{4}(a=0.11918 \AA, \epsilon=0.001 \mathrm{eV}$. $)$

In the description which follows precise account is taken of molecular rotations and translations. All the molecules are assumed to be in their ground vibrational state and zero-point vibrations are ignored. We also assume the molecule is in its ground electronic state which is $a^{3} \Sigma_{g}^{-}$state with spin one.

For unpolarized neutrons the magnetic and nuclear scattering are independent and do not interfere. Only the magnetic cross section is given here. The nuclear contribution was computed using the rigorous quantummechanical method of Lurie ${ }^{5}$ which includes both interference and direct scattering. 


\section{MAGNETIC CROSS SECTION}

The magnetic scattering in $\mathrm{O}_{2}$ results from the interaction between the magnetic moment of the neutron and the molecule. The usual scattering experiment consists of a beam of monoenergetic unpolarized neutrons with wave vector $\mathbf{k}$ incident on a sample of $\mathrm{O}_{2}$ gas in thermal equilibrium. The scattering molecule undergoes a transition from an energy $E_{i}$ to a final energy $E_{f}$, while neutron gains an energy $\epsilon$ and is scattered with a final wave vector $\mathbf{k}^{\prime}$. In the Born approximation the double-differential magnetic-scattering cross section is given by

$$
\begin{array}{r}
\frac{\partial^{2} \sigma^{\mathrm{mag}}}{\partial \mathbf{\Omega} \partial \epsilon}=\frac{k^{\prime}}{k} \frac{4 b^{2}}{3} \int_{-\infty}^{\infty} \frac{d t}{2 \pi \hbar} \exp (i \epsilon t / \hbar)\left\langle\exp \left(i \mathbf{\kappa} \cdot \mathbf{R}_{\mathbf{0}}\right)\right. \\
\left.\times \exp \left[-i \boldsymbol{\kappa} \cdot \mathbf{R}_{\mathbf{0}}(t)\right]\right\rangle_{\boldsymbol{T}}\left\langle F^{*}(\boldsymbol{\kappa}) \cos \left(\boldsymbol{\kappa} \cdot \mathbf{R}^{\prime} / 2\right)\right. \\
\left.\times \cos \left[\boldsymbol{\kappa} \cdot \mathbf{R}^{\prime}(t) / 2\right] F(\boldsymbol{\kappa}, t)\right\rangle_{\boldsymbol{T}},
\end{array}
$$

where $\boldsymbol{k}$ is the wave vector transfer $\mathbf{k}=\mathbf{k}^{\prime}-\mathbf{k}, \mathbf{R}_{\mathbf{0}}$ is the Heisenberg position operator of the center of mass of the oxygen molecule and $R^{\prime} / 2$ is the equilibrium-position operator for the oxygen nucleus with respect to the center of mass, $b$ is the "magnetic-scattering length"

$$
b=5.402 \times 10^{-13} \mathrm{~cm} \text {, }
$$

and $F(\boldsymbol{x})$ is the magnetic form factor for the molecular electronic wavefunctions. In the above, we have ignored the contribution of the orbital angular momentum to the cross section. This is a standard approximation for this molecule. ${ }^{4}$ The form factor, $F(\boldsymbol{r})$, depends upon the molecular orientation and as such does not commute with the rotational Hamiltonian.

If we take the simplest molecular orbital ${ }^{4,6}$ to compute the magnetic form factor and neglect the overlap terms,

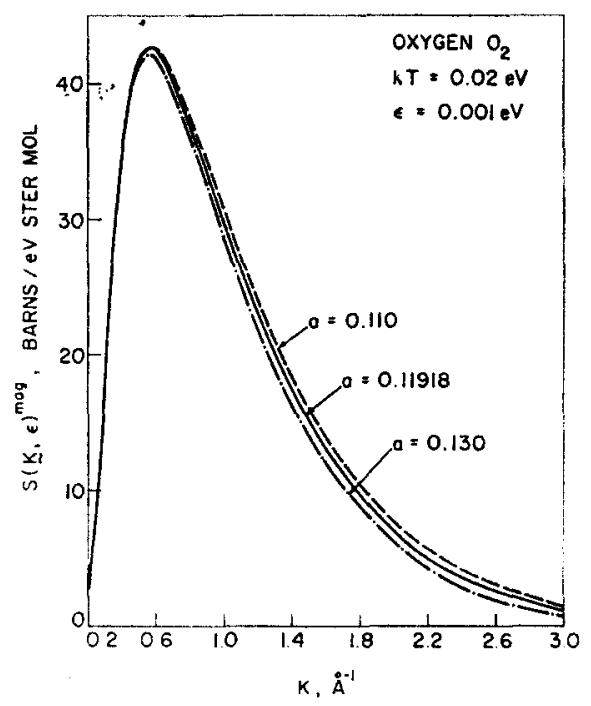

FIG. 3. Magnetic contributions to the scattering law for gaseous, molecular oxygen for three different values of $a$.

we obtain

$$
\begin{aligned}
& F(\mathrm{k})=F_{1}(\kappa)+F_{2}(\kappa)\left[\left(\boldsymbol{\kappa} \cdot \mathrm{R}^{\prime}\right)^{2} / \kappa^{2} R^{\prime 2}\right], \\
& F_{1}(\kappa)=\left[1 /\left(1+\kappa^{2} a^{2}\right)^{3}\right]-\left[3 \kappa^{2} a^{2} /\left(1+\kappa^{2} a^{2}\right)^{4}\right], \\
& F_{2}(\kappa)=3 \kappa^{2} a^{2} /\left(1+\kappa^{2} a^{2}\right)^{4} .
\end{aligned}
$$

The constant $a$ in this expression is the effective Bohr radius for the antibonding electrons. Kleiner ${ }^{4}$ gives this constant as the Bohr radius for an effective nuclear charge $Z=4.44$. Evaluating the thermal averages in Eq. (1) and performing the Fourier transform gives the result

$$
\begin{aligned}
\frac{\partial^{2} \sigma^{\mathrm{mag}}}{\partial \boldsymbol{\Omega} \partial \epsilon}= & \sum_{\nu \mu} \frac{1}{3} b^{2} \frac{k^{\prime}}{k}\left(\frac{\beta}{4 \pi E_{R}}\right)^{1 / 2} Z^{-1} \sum_{J}(2 J+1) \exp \left(-\beta E_{J}\right) \sum_{J^{\prime}} \exp \left[-\left(\epsilon+E_{J^{\prime}}-E_{J}+E_{R}\right)^{2} \frac{\beta}{4 E_{R}}\right] \\
& \times \sum_{l}(2 l+1)\left(2 \delta_{\nu \mu}-1\right)^{l}\left[C_{00^{J}}{ }^{\prime} J^{\prime}\right]^{2}\left\{F_{1} j_{l} j_{l}\left(\kappa R^{\prime} / 2\right)-2 F_{1} F_{2} j_{l}\left(\kappa R^{\prime} / 2\right)\right. \\
& \times\left[j_{l+2}\left(\kappa R^{\prime} / 2\right)-\frac{(2 l+1) j_{l+1}\left(\kappa R^{\prime} / 2\right)}{\kappa R^{\prime} / 2}+\frac{l(l+1) j_{l}\left(\kappa R^{\prime} / 2\right)}{\left(\kappa R^{\prime} / 2\right)^{2}}\right] \\
& \left.+F_{2}^{2}\left[j_{l+2}\left(\kappa R^{\prime} / 2\right)-\frac{(2 l+1) j_{l+1}\left(\kappa R^{\prime} / 2\right)}{\kappa R^{\prime} / 2}+\frac{l(l+1) j_{l}\left(\kappa R^{\prime} / 2\right)}{\left(\kappa R^{\prime} / 2\right)^{2}}\right]^{2}\right\},
\end{aligned}
$$

where

$$
E_{J}=\left(\hbar^{2} / 2 I\right) J(J+1), \quad E_{R}=\hbar^{2} \kappa^{2} / 2 M,
$$

$C_{00}{ }^{\prime l} J^{\prime}$ is a Clebsch-Gordan coefficient, and $j_{l}(x)$ is the spherical Bessel function.

\section{CaLCULATIONS}

Because of the nature of the calculational method used wherein each discrete rotational level is summed, and because so many levels are populated in oxygen at room temperature, it was convenient to perform the

'G. Herzberg, Molecular Spectra and Molecular Siructure (D. Van Nostrand Co., Inc., New York, 1950), Vol. 1, p. 357. 
calculations at a somewhat reduced temperature $(k T=20 \mathrm{meV})$. The restriction was imposed only to reduce the computation time and can easily be removed. Of course the sum in Eq. (3) is carried out only over even values of $J$ and $J^{\prime}$.

The calculations were performed for a value of $a$ corresponding to that used by $\operatorname{Kleiner}^{4}(a=0.11918 \AA)$ and for values somewhat larger $(a=0.13 \AA)$ and smaller $(a=0.11 \AA)$.

The nuclear and magnetic contributions to the scattering law for several energy transfers are compared in Figs. 1 and 2. The scattering law is related to the cross section as follows

$$
S(\boldsymbol{\kappa}, \epsilon)=\left(k / k^{\prime}\right)\left(\partial^{2} \sigma / \partial \boldsymbol{\Omega} \boldsymbol{\partial} \epsilon\right) .
$$

It is apparent from these results that the magnetic contribution to the inelastic scattering is discernable. It is also apparent that there are in fact discernable differences in the magnetic contribution for different moment distributions. However, comparing Figs. 2 and 3 , we see that "reasonable" changes in the moment distribution produce rather small changes in the cross section. As might be expected the cross section is more sensitive to the shape of the moment distribution than to its size.

Parameters used in the calculations were $R^{\prime}=1.21 \AA$, nuclear scattering lengths, $A=0.58 \times 10^{-12} \mathrm{~cm}, C=0$, where $A$ is the coherent and $C$ is the incoherent scattering length.

\section{ACKNOWLEDGMENT}

We wish to thank Professor G. Venkataraman for his advice and counsel during the course of this work. Part of this work was done while one of us (G.C.S.) was at the Idaho Nuclear Corporation.

\title{
van der Waals Potentials for Simple Polar Molecules Interacting with Graphite*
}

\author{
A. D. Crowell \\ Department of Physics, University of Vermont, Burlington, Vermont
}

(Received 20 November 1967)

\begin{abstract}
A simple model for calculating the interaction potentials of simple (nearly spherical) polar molecules with graphite based on a lattice summation of the $(6-12)$ portion of a Stockmayer potential and the effects of a classical dipole image is proposed. Results are obtained and discussed for $\mathrm{NH}_{3}$ and $\mathrm{SO}_{2}$.
\end{abstract}

\section{INTRODUCTION}

Values of the interaction potential energies of simple nonpolar molecules in the neighborhood of graphite surfaces have been calculated by several investigators using lattice sums of (6-12), (6-exp), and more complicated pairwise potentials. ${ }^{1-5}$ The results of such calculations have been compared with experimental isosteric and calorimetric heats of adsorption and with the energies obtained from surface virial effects by Halsey and co-workers. ${ }^{6}$ Quite satisfactory agreement has been obtained in spite of the various rather arbi-

* Supported by U.S. Army Research Office (Durham) Contract No. DA-31-124-ARO(D) -172.

1 A. D. Crowell and D. M. Young, Trans. Faraday Soc. 49, 1080 (1953).

${ }_{2}$ N. N. Avgul, A. A. Isirikyan, A. V. Kiselev, L. A. Lygina, and D. P. Poshkus, Izv. Akad. Nauk SSSR Otd. Khim. Nauk. 1957, 1314.

A. D. Crowell and R. B. Steele, J. Chem. Phys. 34, 1347 (1961).

${ }^{4} \mathrm{~S}$. Ross and J. P. Olivier, On Physical Adsorption (John Wiley \& Sons, Inc., New York, 1964).

5. D. Crowell and C. O. Chang, J. Chem. Phys. 43, 4364 (1965).

${ }^{6}$ W. A. Steele and G. D. Halsey, Jr., J. Chem. Phys. 22, 979 (1954) J. R. Sams, Jr., G. Constabaris, and G. D. Halsey, Jr., J. Phys. Chem. 64, 1689 (1960), and others. trary methods employed in choosing the necessary parameters for the potential functions. The purpose of the present investigation was to extend the techniques developed previously for nonpolar molecules to polar molecules.

The following calculations assume that the permanent dipole moment of a polar molecule induces a classical image dipole in the conduction electrons of graphite. The total interaction of the molecule with the graphite is then assumed to be due to a lattice sum of a pairwise dispersion and repulsive $(6-12)$ potential plus the electrostatic interaction between the dipole and its image. The necessary potential parameters are inferred from the values obtained for polar molecules in the Stockmayer ${ }^{7}$ potential and the (6-12) potential constants previously obtained for graphite.

\section{INTERACTION BETWEEN POLAR MOLECULES AND GRAPHITE}

Using the notations employed earlier, the distance between the center of the polar molecule [the location

\footnotetext{
${ }^{7}$ W. H. Stockmayer, J. Chem. Phys. 9, 398 (1941)
} 